\title{
Wheat Genotypes under Restricted Irrigated Conditions
}

\author{
Rana Saha, Hausila Prasad Singh", Sandeep Kumar, \\ Md. Ekram Hussain and R. S. Shukla
}

\begin{abstract}
Department of Plant Breeding and Genetics, Jawaharlal Nehru Krishi Vishwa Vidyalaya, Jabalpur - 482004, India
\end{abstract}

*Corresponding author

\begin{tabular}{|l|}
\hline Ke y w o r d s \\
$\begin{array}{l}\text { Correlation coefficient, } \\
\text { Genetic variability, } \\
\text { harvesting index and path } \\
\text { coefficient }\end{array}$ \\
\hline Article Info \\
\hline $\begin{array}{l}\text { Accepted: } \\
\text { 22 May } 2018 \\
\text { Available Online: } \\
\text { 10 June } 2018\end{array}$ \\
\hline
\end{tabular}

A B S T R A C T

\begin{abstract}
Wheat genotypes were shown under restricted irrigated condition, based on morphological traits and their genetic variability, correlation coefficient analysis, path coefficient analysis and rank germplasm based on principal component analysis for yield and yield attributing traits. This investigation was carried out with 30 genotypes of wheat using 17 traits viz., days to $50 \%$ flowering, days to maturity, plant height, number of effective tillers per plant, number of ears per plant, number of spikelets per spike, ear length, ear weight, peduncle length, number of grains per ear, 1000-grain weight, grain yield per plant, biological yield per plant, harvest index, relative water content, chlorophyll content and canopy temperature. The high heritability coupled with high genetic advance as percentage of mean was recorded for ear weight, harvest index, biological yield, number of ears per plant, grain yield, number of effective tillers per plant, peduncle length, number of grains per ear, ear length, number of spikelets per spike, total grain weight and canopy temperature. The high heritability coupled with moderate genetic advance was recorded for chlorophyll content and relative water content whereas, high heritability coupled with low genetic advance was observed for plant height, days to $50 \%$ heading and days to maturity. On the basis of correlation and path studies of the present investigation, most important traits identified among the promising genotypes of wheat were biological yield, number of ears per plant and harvest index. On the basis of principal component analysis 5 principal components (PCs) showed about $68.05 \%$ variability and germplasm viz., PYT61, PYT-101, PYT-22, PYT-49, PYT-40, PYT-16, PYT-97, PYT-51, PYT-115 and PYT27 had been identified as promising genotypes as they posses higher values for yield and yield attributing traits.
\end{abstract}

\section{Introduction}

Bread wheat is the world's second most important staple food crop for more than 35 percent of world's population next to the rice. It is cultivated under a wide range of climatic conditions favoured by cool, moist weather followed by dry warm weather. It produces about $20 \%$ food resources of the world. India is the second largest wheat producer in the world next to China with a production level of 95.91 million tonnes (FAO 2015). Despite remarkable growth in food production, the risks were exposed by food crisis in the recent years. Therefore, wheat production must continue to increase by $2 \%$ annually, more 
particularly in developing world including South-East Asia to meet future demands imposed by population and prosperity growth (Reynolds et al., 2008). Accordingly, understanding and improving plant survival and growth under restricted water availability is of central significance in contemporary plant science. This challenge will probably be further compounded by reduced water availability and increased temperature due to global warming. Since, the estimation of genetic parameters like character association and path coefficient serve as an important tool in breeding programmes to improve the concern traits. Simultaneous inheritance of characters that help to decide breeding strategies may vary with environmental conditions and set up of experimental genotypes. Hence, it is essential to conduct studies in different environment with diverse available genotypes. The interaction effects of wheat genotypes with water and temperature stress on grains, days to maturity, plant height, peduncle length, number of grains per ear, number of spikelets per spike, 1000- grain weight, chlorophyll content, canopy temperature and RWC have generally been worked out which were established facts to contribute more for yield under restricted irrigation.

\section{Materials and Methods}

A field experiment was carried out under Wheat Improvement Project Department of Plant Breeding and Genetics at Seed Breeding Farm, College of Agriculture, Jawaharlal Nehru Krishi Vishwa Vidyalaya, Jabalpur under restricted irrigated conditions. The experimental area occupied was quite uniform in respect of topography and fertility. The experimental material consists of 30 wheat genotypes including 3 checks viz., DBW71, DBW107 and K307. The experiment was conducted in three replications under Randomized Complete Block Design. Each plot consisted of six rows of $2.5 \mathrm{~m}$ length and $22.5 \mathrm{~cm}$. Details of genotypes are listed in table 1.

\section{Correlation coefficient}

Correlation coefficients were calculated for a combination of all characters under study at genotypic, phenotypic and environmental levels with the help of formula suggested by Miller et al., (1958). To test the significance of phenotypic correlation coefficients, the estimated values were compared with the tabulated values of Fisher and Yates (1963) at n-2 d.f. at two levels of probability, viz., 5\% and $1 \%$.

\section{Path coefficient analysis}

Path coefficient is a standardized partial regression, which measures the direct influence of one variable upon another and allows partition of correlation coefficient into components of direct and indirect effects. The proportion of direct and indirect contributions of various characteristics to the total correlation coefficients with seed yield was estimated through path coefficient analysis as suggested by Wright (1921) and elaborated by Dewey and Lu (1959). To estimate various direct and indirect effects, the sets of simultaneous equations were formed and solved for estimating direct and indirect effects. Residual effect measures the contribution of the characters not considered in the causal scheme.

\section{Results and Discussion}

\section{Correlation coefficient}

The phenotypic and genotypic correlation coefficients for different pairs of character were studied and results are presented in table 2 and 3. The correlation coefficients were tested for their significance at (n-2) degree of 
freedom. In general, the result showed that genotypic correlation coefficients were lower than phenotypic correlation coefficient for almost all the characters under study, which were in turn higher than the environmental correlation.

\section{Days to $50 \%$ heading}

Days to $50 \%$ flowering had a highly significant positive correlation with days to maturity (0.7299), chlorophyll content (0.2959) and harvest index (0.2924). Association of days to $50 \%$ heading with other traits studied were very low in magnitude and non-significant.

\section{Days to maturity}

Days to maturity had highly significant positive correlation with grain yield per plant (0.4706), number of grains per ear (0.4467), number of spikelets per ear (0.4164), biological yield (0.3513), and 1000 grain weight (0.3121). Significant positive correlation with ear weight (0.2428). Whereas, RWC (-0.2363) had a significant negative effect on days to maturity. Association of days to maturity with other traits studied were very low in magnitude and non-significant.

\section{Plant height}

Plant height had a highly significant positive correlation with peduncle length (0.7973), ear weight (0.6898), ear weight (0.6668), number of spikelets per spike (0.2969) and significant positive correlation with canopy temperature (0.2258). Association of plant height with other traits studied were very low in magnitude and non-significant.

\section{Peduncle length}

Ear weight (0.8617), ear length (0.8584), number of spikelets per spike (0.5918), canopy temperature $(0.3378)$ number of grains per ear (0.3375) had a positive highly significant correlation with peduncle length. Grain yield per plant (0.2926) and biological yield (0.2305) had positive significant with peduncle length. Association of peduncle height with other traits studied was very low in magnitude and non-significant.

\section{Ear length}

Ear length had a highly significant positive correlation with ear weight (0.9652), number of spikelets per spike (0.6515), number of grains per ear (0.4560), canopy temperature (0.3406) and grain yield per plant (0.3240). Harvest index (0.2309) had positive significant with ear length. Association of ear length with other traits studied were very low in magnitude and non-significant.

\section{Ear weight}

Number of spike lets per spike (0.696), number of grains per ear (0.4148), grain yield per plant (0.3318) and canopy temperature (0.3063) had positive highly significant correlation with ear weight. Harvest index (0.2193) had positive significant with ear weight.

Association of ear weight with other traits studied were very low in magnitude and nonsignificant.

\section{Number of effective tillers per plant}

Number of effective tillers per plant had positive highly significant with a number of ears per plant (0.9952) and positive significant with (0.2574). Harvest index (-0.2399) had negative significant with a number of effective tillers per plant. Association of a number of effective tillers per plant with other traits studied were very low in magnitude and nonsignificant. 


\section{Number of ears per plant}

Number of ears per plant had positive significant with biological yield (0.2619) and negative significant with harvest index (0.2420). Association of a number of ear per plant with other traits studied were very low in magnitude and non-significant.

\section{Number of spikelets per spike}

Number of spikelets per spike had a positive highly significant correlation with the number of grains per ear (0.7438), grain yield per plant (0.5823), 1000 grain weight (0.5213), canopy temperature (0.4622) and biological yield (0.3741). Association of a number of spikelets per spike with other traits studied were very low in magnitude and non-significant.

\section{Number of grains per ear}

Number of grains per ear had a positive highly significant correlation with grain yield per plant (0.7985), 1000 grain weight (0.6893), biological yield (0.4821) and canopy temperature (0.3230). Association of a number of grains per ear with other traits studied were very low in magnitude and non-significant.

\section{0 grain weight}

Grain yield per plant (0.8323), Harvest index $(0.3624)$ and biological yield $(0.3608)$ had positive highly significant correlation with 1000 grain weight. Association of 1000- grain weight with other traits studied were very low in magnitude and non-significant.

\section{Biological yield}

Grain yield per plant (0.5526) had a positive highly significant correlation with biological yield and had negative highly significant with harvest index (-0.6085) and RWC (-0.3583) had negative highly significant with biological yield. Association of biological yield with other traits studied were very low in magnitude and non-significant.

\section{Harvest index}

Harvest index had positive highly significant with 1000 grain weight $(0.3624)$, grain yield per plant (0.3002), days to $50 \%$ heading (0.2924), and positive significant with ear length (0.2309), ear weight (0.2193), and negative highly significant with biological yield $(-0.6085)$ and negative significant with number of ears per plant (-0.2420), number of effective tillers per plant (-0.2399). Association of harvest index with other traits studied were very low in magnitude and nonsignificant.

\section{Chlorophyll content}

Chlorophyll content had positive highly significant with days to $50 \%$ heading (0.2959). Association of chlorophyll content with other traits studied was very low in magnitude and non-significant.

\section{Canopy temperature}

Canopy temperature had positive highly significant with a number of spikelets per spike (0.4622), ear length (0.3406), peduncle length (ear length), number of grains per ear (0.3230), ear weight $(0.3063)$ and positive significant with plant height (0.2258). Association of canopy temperature with other traits studied was very low in magnitude and non-significant.

\section{Relative water content}

Relative water content had a positive highly significant correlation with biological yield (0.3588) and negative highly significant with days to maturity (-0.2363) and negative significant with grain yield per plant (- 
0.2141). Associations of RWC with other traits studied were very low in magnitude and non-significant.

\section{Path coefficient analysis}

Path coefficients are standardized partial regression coefficients. It partitions the observed correlation between dependent and independent variable in direct and indirect effects in such a manner that observed a correlation between dependent and independent trait is equal to the sum of direct and all possible indirect effects. Path coefficient analysis, which measures the direct and indirect effect of one variable through another on the end product, was carried out using genotypic as well as phenotypic correlation considering grain yield per plant as a dependent variable. The observed genotypic and phenotypic correlation coefficient on grain and its components were partitioned into direct and indirect effects and have been presented in table 4 and 5.

\section{Direct effects}

Biological yield revealed highest direct positive effect (0.9249) on grain yield followed by number of ears per plant (0.8992), harvest index (0.8695), ear length (0.3532), 1000 grain yield $(0.2045)$, days to maturity (0.1848), chlorophyll content (0.0738), peduncle length (0.0689), canopy temperature (0.0033). However, number of effective tillers per plant (-0.8378), ear weight (-0.3400), days to $50 \%$ heading $(-0.2169)$, plant height ($0.0816)$, number of grains per ear $(-0.0731)$, RWC (-0.0547) and number of spikelets per spike (-0.0229) had direct negative effect on grain yield.

\section{Indirect effect}

\section{Days to 50\% heading}

The association of days to $50 \%$ flowering with grain yield per plant was positive and small (0.0973) and it exerted positive indirect effect via RWC (0.0381), canopy temperature (0.0259) on grain yield per plant. Days to $50 \%$ flowering showed negative indirect effect via days to maturity (-0.1599), chlorophyll content (-0.0669). Other indirect effects of this trait via other characters were relatively low and negligible.

\section{Days to maturity}

Days to maturity exhibited a moderate positive direct effect on grain yield per plant (0.4882). The positive indirect effect exerted on grain yield by other characters via days to $50 \%$ heading (0.1362), number of grains of the ear (0.855), 1000 grain weight (0.0671), biological yield (0.0609) and negative indirect effect exerted on RWC (-0.0452), canopy temperature (-0.0051). Other indirect effects of this trait via other characters were relatively low and negligible

Table.1 The details of 30 wheat genotypes

\begin{tabular}{|c|c|c|c|c|c|}
\hline SI.no. & Lines & Lines & Sl.no. & Lines \\
\hline 1 & PYT-2 & 11 & PYT-46 & 21 & PYT-69 \\
\hline \hline 2 & PYT-6 & 12 & PYT-47 & 22 & PYT-89 \\
\hline 3 & PYT-16 & 13 & PYT-49 & 23 & PYT-97 \\
\hline 4 & PYT-21 & 14 & PYT-51 & 24 & PYT-101 \\
\hline 5 & PYT-22 & 15 & PYT-57 & 25 & PYT-104 \\
\hline 6 & PYT-23 & 16 & PYT-58 & 27 & PYT-109 \\
\hline 7 & PYT-24 & 17 & PYT-60 & 28 & PYT-115 \\
\hline 8 & PYT-25 & 18 & PYT-61 & 29 & PYT-118 \\
\hline 9 & PYT-27 & 19 & PYT-66 & 30 & PYT-120 \\
\hline 10 & PYT-40 & 20 & PYT-68 & PYT-122 \\
\hline
\end{tabular}


Table.2 Phenotypic correlation coefficient analysis for yield and its component traits

\begin{tabular}{|c|c|c|c|c|c|c|c|c|c|c|c|c|c|c|c|c|c|c|}
\hline SL. N0 & Characters & DFF & DM & PH & PL & EL & EW & NETPP & NEPP & NSPS & NGPE & TGW & BY & HI & $\mathrm{CC}$ & CT & RWC & GYPP \\
\hline 1 & DFF & 1.000 & $0.729 * *$ & 0.082 & 0.004 & -0.005 & 0.051 & 0.009 & 0.002 & 0.057 & 0.050 & 0.080 & -0.107 & $0.292 * *$ & $0.295^{* *}$ & -0.127 & -0.169 & 0.089 \\
\hline 2 & DM & & 1.000 & 0.017 & 0.160 & 0.206 & $0.242^{*}$ & 0.101 & 0.105 & $0.416^{* *}$ & $0.446 * *$ & $0.312 * *$ & $0.351 * *$ & 0.078 & 0.082 & -0.032 & $-0.236^{*}$ & $0.470^{* *}$ \\
\hline 3 & PH & & & 1.000 & $0.797^{* *}$ & $0.666^{* *}$ & $0.689^{* *}$ & -0.139 & -0.165 & $0.296^{* *}$ & 0.027 & 0.064 & 0.081 & 0.097 & 0.198 & $0.225^{*}$ & 0.025 & 0.103 \\
\hline 4 & PL & & & & 1.000 & $0.858^{* *}$ & $0.861^{* *}$ & -0.183 & -0.198 & $0.591^{* *}$ & $0.337^{* * *}$ & 0.145 & $0.230^{*}$ & 0.085 & -0.021 & $0.337^{* *}$ & 0.043 & $0.292 * *$ \\
\hline 5 & EL & & & & & 1.000 & $0.965^{* *}$ & -0.072 & -0.084 & $0.651^{* *}$ & $0.456^{* *}$ & 0.178 & 0.105 & $0.230^{*}$ & -0.049 & $0.340 * *$ & 0.126 & $0.324 * *$ \\
\hline 6 & EW & & & & & & 1.000 & -0.081 & -0.090 & $0.629 * *$ & $0.414 * *$ & 0.193 & 0.131 & $0.219^{*}$ & 0.004 & $0.306^{* *}$ & 0.118 & $0.331^{* *}$ \\
\hline 7 & NETPP & & & & & & & 1.000 & $0.995^{* * *}$ & 0.060 & 0.134 & -0.012 & $0.257^{*}$ & $-0.239^{*}$ & -0.115 & -0.101 & -0.161 & 0.092 \\
\hline 8 & NEPP & & & & & & & & 1.000 & 0.060 & 0.143 & -0.004 & $0.261 *$ & $-0.242^{*}$ & -0.134 & -0.099 & -0.157 & 0.098 \\
\hline 9 & NSPS & & & & & & & & & 1.000 & $0.743^{* *}$ & $0.521 * *$ & $0.374 * *$ & 0.125 & -0.152 & $0.462 * *$ & -0.115 & $0.582^{* * *}$ \\
\hline 10 & NGPE & & & & & & & & & & 1.000 & $0.689 * *$ & $0.482 * *$ & 0.193 & -0.119 & $0.323 * *$ & -0.191 & $0.798^{* * *}$ \\
\hline 11 & TGW & & & & & & & & & & & 1.000 & $0.360 * *$ & $0.362^{* *}$ & -0.097 & 0.165 & -0.062 & $0.832^{* * *}$ \\
\hline 12 & BY & & & & & & & & & & & & 1.000 & $-0.608^{* *}$ & -0.046 & 0.107 & $-0.358^{* *}$ & $0.552 * *$ \\
\hline 13 & HI & & & & & & & & & & & & & 1.000 & 0.109 & -0.034 & 0.194 & $0.300^{* *}$ \\
\hline 14 & $\mathrm{CC}$ & & & & & & & & & & & & & & 1.000 & -0.135 & 0.075 & 0.002 \\
\hline 15 & CT & & & & & & & & & & & & & & & 1.000 & 0.059 & 0.100 \\
\hline 16 & RWC & & & & & & & & & & & & & & & & 1.000 & $-0.214^{*}$ \\
\hline 17 & GYPP & & & & & & & & & & & & & & & & & 1.000 \\
\hline
\end{tabular}

DFF-Days to 50\% flowering, DM-Days to maturity, PH-Plant height, PL-Peduncle length, EL- Ear length, EW- Ear weight, NETP-Number of effective tillers per plant, NEPP-Number of ears per plant, NSPS-Number of spikelets per spike, NGPE-Number of grains per ear, TGW-Thousand grain weight, BYPPBiological yield per plant, HI-Harvest index, GYPP-Grain yield per plant, CC-Chlorophyll content, CT-Canopy temperature, RWC-Relative water content. 
Table.3 Genotypic path coefficient analysis for yield and its component traits

\begin{tabular}{|c|c|c|c|c|c|c|c|c|c|c|c|c|c|c|c|c|c|}
\hline & $\mathrm{x1}$ & $\mathrm{x} 2$ & $x 3$ & $\mathrm{x} 4$ & $\mathrm{x} 5$ & x6 & $x 7$ & $\mathrm{x8}$ & x9 & x10 & $\mathrm{x12}$ & $\mathrm{x} 13$ & x14 & x15 & x16 & $\mathbf{x 1 7}$ & X11 \\
\hline $\mathbf{x 1}$ & -0.2169 & -0.1599 & -0.0196 & -0.0015 & -0.0007 & -0.0123 & -0.0009 & 0.0002 & -0.0130 & -0.0113 & -0.0187 & 0.0239 & -0.0660 & -0.0669 & 0.0259 & 0.0381 & 0.0973 \\
\hline $\mathbf{x 2}$ & 0.1362 & 0.1848 & 0.0056 & 0.0305 & 0.0413 & 0.0474 & 0.0187 & 0.0204 & 0.0795 & 0.0855 & 0.0609 & 0.0671 & 0.0147 & 0.0158 & -0.0051 & -0.0452 & 0.4882 \\
\hline $\mathbf{x} 3$ & -0.0074 & -0.0025 & -0.0816 & -0.0659 & -0.0561 & -0.0583 & 0.0117 & 0.0140 & -0.0248 & -0.0022 & -0.0052 & -0.0067 & -0.0080 & -0.0164 & -0.0187 & -0.0021 & 0.1053 \\
\hline $\mathrm{x} 4$ & 0.0005 & 0.0114 & 0.0556 & 0.0689 & 0.0604 & 0.0610 & -0.0128 & -0.0137 & 0.0411 & 0.0233 & 0.0099 & 0.0159 & 0.0059 & -0.0015 & 0.0237 & 0.0030 & 0.2934 \\
\hline $\mathrm{x5}$ & 0.0012 & 0.0789 & 0.2428 & 0.3099 & 0.3532 & 0.3484 & -0.0247 & -0.0293 & 0.2341 & 0.1636 & 0.0652 & 0.0373 & 0.0841 & -0.0180 & 0.1241 & 0.0459 & 0.3306 \\
\hline x6 & -0.0192 & -0.0872 & -0.2428 & -0.3011 & -0.3354 & -0.3400 & 0.0256 & 0.0308 & -0.2192 & -0.1439 & -0.0701 & -0.0454 & -0.0766 & -0.0004 & -0.1052 & -0.0413 & 0.3380 \\
\hline $\mathbf{x} 7$ & -0.0036 & -0.0849 & 0.1196 & 0.1558 & 0.0587 & 0.0630 & -0.8378 & -0.8379 & -0.0519 & -0.1129 & 0.0133 & -0.2168 & 0.2045 & 0.0969 & 0.0835 & 0.1372 & 0.0917 \\
\hline x8 & -0.0007 & 0.0991 & -0.1540 & -0.1792 & -0.0746 & -0.0815 & 0.8993 & 0.8992 & 0.0575 & 0.1303 & -0.0061 & 0.2376 & -0.2213 & -0.1216 & -0.0885 & -0.1432 & 0.0989 \\
\hline x9 & -0.0014 & -0.0098 & -0.0069 & -0.0137 & -0.0152 & -0.0147 & -0.0014 & -0.0015 & -0.0229 & -0.0171 & -0.0120 & -0.0086 & -0.0029 & 0.0035 & -0.0107 & 0.0027 & 0.5880 \\
\hline $\mathbf{x 1 0}$ & -0.0038 & -0.0338 & -0.0020 & -0.0248 & -0.0339 & -0.0310 & -0.0099 & -0.0106 & -0.0546 & -0.0731 & -0.0507 & -0.0353 & -0.0142 & 0.0088 & -0.0239 & 0.0140 & 0.8017 \\
\hline x12 & 0.0176 & 0.0674 & 0.0129 & 0.0295 & 0.0378 & 0.0422 & -0.0032 & -0.0014 & 0.1075 & 0.1416 & 0.2045 & 0.0741 & 0.0745 & -0.0200 & 0.0351 & -0.0129 & 0.8397 \\
\hline $\mathrm{x} 13$ & -0.1018 & 0.3358 & 0.0762 & 0.2138 & 0.0977 & 0.1235 & 0.2393 & 0.2444 & 0.3485 & 0.4463 & 0.3351 & 0.9249 & -0.5640 & -0.0432 & 0.1007 & -0.3318 & 0.5543 \\
\hline x14 & 0.2647 & 0.0693 & 0.0854 & 0.0740 & 0.2072 & 0.1958 & -0.2122 & -0.2140 & 0.1096 & 0.1688 & 0.3166 & -0.5303 & 0.8695 & 0.0951 & -0.0310 & 0.1698 & 0.2973 \\
\hline x15 & 0.0227 & 0.0063 & 0.0148 & -0.0016 & -0.0038 & 0.0001 & -0.0085 & -0.0100 & -0.0114 & -0.0089 & -0.0072 & -0.0034 & 0.0081 & 0.0738 & -0.0102 & 0.0057 & 0.0013 \\
\hline x16 & -0.0004 & -0.0001 & 0.0008 & 0.0011 & 0.0012 & 0.0010 & -0.0003 & -0.0003 & 0.0016 & 0.0011 & 0.0006 & 0.0004 & -0.0001 & -0.0005 & 0.0033 & 0.0002 & 0.0997 \\
\hline $\mathbf{x 1 7}$ & 0.0096 & 0.0134 & -0.0014 & -0.0024 & -0.0071 & -0.0067 & 0.0090 & 0.0087 & 0.0064 & 0.0105 & 0.0035 & 0.0196 & -0.0107 & -0.0042 & -0.0033 & -0.0547 & -0.2149 \\
\hline
\end{tabular}

Residual effect $(r)=0.024$

Bold diagonal values are direct effects of each independent trait.

Off diagonal values are indirect effects of each dependent trait via independent traits.

X1-Days to 50\% heading, X2-Days to maturity, X3-Plant height, X4-Peduncle length, X5-Ear length, X6-Ear weight, X7-Number of effective tillers per pant,

X8-Number of ears per plant, X9-Number of spikelets per spike, X10-Number of grain per ear, X11-Grain yield, X12-Thousand grain weight, X13-Biological yield, X14-Harvest index, X15-Chlorophyll content, X16- Canopy temperature, X17- Relative water content 
Table.4 Phenotypic path coefficient analysis for yield and its traits

\begin{tabular}{|c|c|c|c|c|c|c|c|c|c|c|c|c|c|c|c|c|c|}
\hline & x1 & x2 & x3 & $\mathrm{x} 4$ & $\mathrm{x} 5$ & x6 & $\mathbf{x} 7$ & $\mathrm{x} 8$ & x9 & x10 & x12 & x13 & x14 & x15 & x16 & x17 & X11 \\
\hline $\mathbf{x 1}$ & -0.2123 & -0.1550 & -0.0176 & -0.0010 & 0.0001 & -0.0108 & -0.0020 & -0.0004 & -0.0121 & -0.0106 & -0.0171 & 0.0227 & -0.0621 & -0.0628 & 0.0270 & 0.0359 & 0.0893 \\
\hline $\mathbf{x} 2$ & 0.1244 & 0.1704 & 0.0030 & 0.0273 & 0.0351 & 0.0414 & 0.0172 & 0.0180 & 0.0709 & 0.0761 & 0.0532 & 0.0599 & 0.0133 & 0.0140 & -0.0055 & -0.0403 & 0.4706 \\
\hline $\mathbf{x} 3$ & -0.0060 & -0.0013 & -0.0730 & -0.0582 & -0.0486 & -0.0503 & 0.0102 & 0.0121 & -0.0217 & -0.0020 & -0.0047 & -0.0059 & -0.0071 & -0.0145 & -0.0165 & -0.0018 & 0.1039 \\
\hline $\mathrm{x} 4$ & 0.0001 & 0.0042 & 0.0209 & 0.0263 & 0.0226 & 0.0226 & -0.0048 & -0.0052 & 0.0155 & 0.0089 & 0.0038 & 0.0061 & 0.0023 & -0.0006 & 0.0089 & 0.0012 & 0.2926 \\
\hline $\mathrm{x5}$ & 0.0000 & 0.0126 & 0.0409 & 0.0526 & 0.0613 & 0.0592 & -0.0045 & -0.0052 & 0.0400 & 0.0280 & 0.0109 & 0.0064 & 0.0142 & -0.0030 & 0.0209 & 0.0078 & 0.3240 \\
\hline x6 & -0.0034 & -0.0163 & -0.0462 & -0.0578 & -0.0647 & -0.0670 & 0.0054 & 0.0061 & -0.0422 & -0.0278 & -0.0130 & -0.0088 & -0.0147 & 0.0000 & -0.0205 & -0.0080 & 0.3318 \\
\hline $\mathbf{x} 7$ & 0.0012 & 0.0130 & -0.0179 & -0.0235 & -0.0093 & -0.0104 & 0.1281 & 0.1275 & 0.0078 & 0.0172 & -0.0016 & 0.0330 & -0.0307 & -0.0148 & -0.0130 & -0.0207 & 0.0927 \\
\hline x8 & -0.0002 & -0.0088 & 0.0139 & 0.0166 & 0.0071 & 0.0076 & -0.0835 & -0.0839 & -0.0051 & -0.0121 & 0.0004 & -0.0220 & 0.0203 & 0.0112 & 0.0083 & 0.0132 & 0.0981 \\
\hline x9 & -0.0007 & -0.0052 & -0.0037 & -0.0074 & -0.0081 & -0.0079 & -0.0008 & -0.0008 & -0.0125 & -0.0093 & -0.0065 & -0.0047 & -0.0016 & 0.0019 & -0.0058 & 0.0014 & 0.5823 \\
\hline $\mathbf{x 1 0}$ & -0.0004 & -0.0040 & -0.0002 & -0.0030 & -0.0041 & -0.0037 & -0.0012 & -0.0013 & -0.0066 & -0.0089 & -0.0061 & -0.0043 & -0.0017 & 0.0011 & -0.0029 & 0.0017 & 0.7985 \\
\hline x12 & 0.0111 & 0.0430 & 0.0089 & 0.0200 & 0.0246 & 0.0267 & -0.0017 & -0.0006 & 0.0719 & 0.0951 & 0.1379 & 0.0498 & 0.0500 & -0.0135 & 0.0229 & -0.0086 & 0.8323 \\
\hline x13 & -0.1029 & 0.3375 & 0.0781 & 0.2214 & 0.1010 & 0.1264 & 0.2473 & 0.2516 & 0.3594 & 0.4632 & 0.3467 & 0.9607 & -0.5846 & -0.0449 & 0.1035 & -0.3442 & 0.5526 \\
\hline x14 & 0.2647 & 0.0708 & 0.0884 & 0.0777 & 0.2091 & 0.1985 & -0.2172 & -0.2191 & 0.1136 & 0.1755 & 0.3281 & -0.5509 & 0.9053 & 0.0990 & -0.0308 & 0.1760 & 0.3002 \\
\hline x15 & 0.0099 & 0.0028 & 0.0067 & -0.0007 & -0.0016 & 0.0000 & -0.0039 & -0.0045 & -0.0051 & -0.0040 & -0.0033 & -0.0016 & 0.0037 & $\mathbf{0 . 0 3 3 5}$ & -0.0045 & 0.0025 & 0.0029 \\
\hline x16 & -0.0014 & -0.0003 & 0.0024 & 0.0036 & 0.0036 & 0.0033 & -0.0011 & -0.0011 & 0.0049 & 0.0034 & 0.0018 & 0.0011 & -0.0004 & -0.0014 & 0.0106 & 0.0006 & 0.1007 \\
\hline $\mathbf{x 1 7}$ & 0.0052 & 0.0073 & -0.0008 & -0.0014 & -0.0039 & -0.0037 & 0.0050 & 0.0048 & 0.0036 & 0.0059 & 0.0019 & 0.0110 & -0.0060 & -0.0023 & -0.0018 & -0.0308 & -0.2141 \\
\hline
\end{tabular}

Residual effect $(r)=0.107$

Bold diagonal values are direct effects of each independent trait.

Off diagonal values are indirect effects of each dependent trait via independent traits.

X1-Days to 50\% heading, X2-Days to maturity, X3-Plant height, X4-Peduncle length, X5-Ear length, X6-Ear weight, X7-Number of effective tillers per pant,

X8-Number of ears per plant, X9-Number of spikelets per spike, X10-Number of grain per ear, X11-Grain yield, X12-Thousand grain weight, X13-Biological yield, X14-Harvest index, X15-Chlorophyll content, X16-Canopy temperature, X17- Relative water content 
Table.5 Genotypic correlation coefficient analysis for yield and its contributing traits

\begin{tabular}{|c|c|c|c|c|c|c|c|c|c|c|c|c|c|c|c|c|c|c|}
\hline $\begin{array}{l}\text { SI. } \\
\text { N0. }\end{array}$ & Characters & DFF & DM & PH & PL & EL & EW & NETPP & NEPP & NSPS & NGPE & TGW & BY & HI & $\mathrm{CC}$ & $\mathrm{CT}$ & RWC & GYPP \\
\hline 1 & DFF & 1.000 & 0.737 & 0.090 & 0.007 & 0.003 & 0.056 & 0.004 & -0.003 & 0.060 & 0.051 & 0.086 & -0.110 & 0.304 & 0.308 & -0.119 & -0.175 & 0.097 \\
\hline 2 & DM & & 1.000 & 0.030 & 0.165 & 0.223 & 0.256 & 0.101 & 0.110 & 0.430 & 0.462 & 0.329 & 0.363 & 0.079 & 0.085 & -0.027 & -0.244 & 0.488 \\
\hline 3 & PH & & & 1.000 & 0.807 & 0.687 & 0.714 & -0.142 & -0.171 & 0.303 & 0.027 & 0.063 & 0.082 & 0.098 & 0.201 & 0.229 & 0.025 & 0.105 \\
\hline 4 & PL & & & & 1.000 & 0.877 & 0.885 & -0.185 & -0.199 & 0.596 & 0.338 & 0.144 & 0.231 & 0.085 & -0.022 & 0.343 & 0.044 & 0.293 \\
\hline 5 & EL & & & & & 1.000 & 0.986 & -0.070 & -0.083 & 0.662 & 0.463 & 0.184 & 0.105 & 0.238 & -0.050 & 0.351 & 0.129 & 0.330 \\
\hline 6 & EW & & & & & & 1.000 & -0.075 & -0.090 & 0.644 & 0.423 & 0.206 & 0.133 & 0.225 & 0.001 & 0.309 & 0.121 & 0.338 \\
\hline 7 & NETPP & & & & & & & 1.000 & 1.000 & 0.061 & 0.134 & -0.015 & 0.258 & -0.244 & -0.115 & -0.099 & -0.163 & 0.091 \\
\hline 8 & NEPP & & & & & & & & 1.000 & 0.064 & 0.145 & -0.006 & 0.264 & -0.246 & -0.135 & -0.098 & -0.159 & 0.098 \\
\hline 9 & NSPS & & & & & & & & & 1.000 & 0.746 & 0.525 & 0.376 & 0.126 & -0.154 & 0.467 & -0.116 & 0.588 \\
\hline 10 & NGPE & & & & & & & & & & 1.000 & 0.692 & 0.482 & 0.194 & -0.120 & 0.326 & -0.191 & 0.801 \\
\hline 11 & TGW & & & & & & & & & & & 1.000 & 0.362 & 0.364 & -0.097 & 0.171 & -0.063 & 0.839 \\
\hline 12 & BY & & & & & & & & & & & & 1.000 & -0.609 & -0.046 & 0.108 & -0.358 & 0.554 \\
\hline 13 & HI & & & & & & & & & & & & & 1.000 & 0.109 & -0.035 & 0.195 & 0.297 \\
\hline 14 & $\mathrm{CC}$ & & & & & & & & & & & & & & 1.000 & -0.138 & 0.076 & 0.001 \\
\hline 15 & CT & & & & & & & & & & & & & & & 1.000 & 0.060 & 0.099 \\
\hline 16 & RWC & & & & & & & & & & & & & & & & 1.000 & -0.214 \\
\hline 17 & GYPP & & & & & & & & & & & & & & & & & 1.000 \\
\hline
\end{tabular}

DFF-Days to 50\% flowering, DM-Days to maturity, PH-Plant height, PL-Peduncle length, EL- Ear length, EW- Ear weight, NETP-Number of effective tillers per plant, NEPP-Number of ears per plant, NSPS-Number of spikelets per spike, NGPE-Number of grains per ear, TGW-Thousand grain weight, BYPPBiological yield per plant, HI-Harvest index, GYPP-Grain yield per plant, CC-Chlorophyll content, CT-Canopy temperature, RWC-Relative water content. 


\section{Plant height}

Plant height exhibited a moderate positive effect on grain yield per plant (0.1053). The positive indirect effect exerted on grain yield by other characters via numbers of ears per plant (0.0140), a number of effective tillers per plant (0.0117) and negative indirect effect exerted on peduncle length (-0.0659), ear weight $(-0.0583)$, ear length $(-0.0561)$. Other indirect effects of this trait via other characters were relatively low and negligible

\section{Peduncle length}

The association of peduncle length with grain yield per plant was positive and small (0.2934) and it exerted positive indirect effect via ear weight (0.0610), ear length (0.0604) and negative indirect effect via number of ears per plant $(-0.0137)$, number of effective tillers per plant (-0.0128). Other indirect effects of this trait via other characters were relatively low and negligible.

\section{Ear length}

Ear length exhibited a moderate positive effect on grain yield per plant (0.3306). The positive indirect effect exerted on grain yield by other characters via ear weight (0.3484), peduncle length (0.3099), plant height (0.2428) and negative indirect effect via numbers of ears per plant (-0.0293), a number of effective tillers per plant (-0.0247). Other indirect effects of this trait via other characters were relatively low and negligible.

\section{Ear weight}

The indirect effect of ear weight on grain yield per plant was positive (0.3380) and it exerted positive indirect effect via number of ears per plant (0.0308), number of effective tillers per plant (0.0256) and negative indirect effect via ear length (-0.3354), plant height (-
0.2428). Other indirect effects of this trait via other characters were relatively low and negligible.

\section{Number of effective tillers per plant}

The indirect effect of Number of effective tillers per plant on grain yield per plant was positive (0.0917) and it exerted positive indirect effect via peduncle length (0.1558), harvest index (0.2045), RWC (0.1372) and negative indirect effect via number of ears per plant (-0.8379), number of grains per ear (0.1129 ). Other indirect effects of this trait via other characters were relatively low and negligible.

\section{Number of ears per plant}

The indirect effect of Number of ears per plant on grain yield per plant was positive (0.0989) and it exerted positive indirect effect via number of effective tillers per plant (0.8993), biological yield (0.2376), number of grains per ear (0.1303) and negative indirect effect via harvest index (-0.2213), peduncle length (-0.1792), plant height (-0.1540). Other indirect effects of this trait via other characters were relatively low and negligible.

\section{Number of spikelets per spike}

The indirect effect of Number of effective tillers per plant on grain yield per plant was positive (0.5880) and it exerted positive indirect effect via chlorophyll content (0.0035), RWC (0.0027) and negative indirect effect via number of grains per ear (-0.0171), ear length (-0.0152). Other indirect effects of this trait via other characters were relatively low and negligible.

\section{Number of grains per ear}

The indirect effect of Number of effective tillers per plant on grain yield per plant was 
positive (0.8017) and it exerted positive indirect effect via RWC (0.0140) and negative indirect effect via a number of spikelets per spike (-0.0546), 1000 grain weight (-0.0507). Other indirect effects of this trait via other characters were relatively low and negligible.

\section{0 grain weight}

The indirect effect of 1000 grain weight on grain yield per plant was positive (0.8397) and it exerted positive indirect effect via harvest index (0.0745), biological yield (0.0741), days to maturity (0.0674) and negative indirect effect via chlorophyll content (-0.0200) and RWC (-0.0129).

\section{Biological yield}

The indirect effect of biological yield on grain yield per plant was positive (0.5543) and it exerted positive indirect effect via number of grains per ear (0.4463), number of spikelets per spike (0.3485), days to maturity (0.3358) and negative indirect effect via harvest index (-0.5640), RWC (-0.3318), days to $50 \%$ heading (-0.1018).

\section{Harvest index}

The indirect effect of biological yield on grain yield per plant was positive (0.2973) and it exerted positive indirect effect via 1000 grain weight (0.3166), ear length (0.2072), ear weight (0.1958) and negative indirect effect via biological yield $(-0.5303)$, number of ears per plant (-0.2140), number of effective tillers per plant $(-0.2144)$.

\section{Chlorophyll content}

Indirect effect of biological yield on grain yield per plant was positive (0.0013) and it exerted positive indirect effect via days to $50 \%$ heading (0.0227), plant height (0.0148), and negative indirect effect via a number of spikelets per spike (-0.0114), canopy temperature (-0.0102). Other indirect effects of this trait via other characters were relatively low and negligible.

\section{Canopy temperature}

Indirect effect of biological yield on grain yield per plant was positive (0.0997) and it exerted positive indirect effect via ear length (0.0012), peduncle length (0.0011) and negative indirect effect via chlorophyll content (-0.0005). Other indirect effects of this trait via other characters were relatively low and negligible.

\section{Relative water content}

Indirect effect of biological yield on grain yield per plant was negative $(-0.2149)$ and it exerted positive indirect effect via biological yield (0.0196), days to maturity (0.0134) and negative indirect effect via harvest index ($0.0107)$, ear length (-0.0071). Other indirect effects of this trait via other characters were relatively low and negligible.

\section{Correlation coefficient analysis}

Correlation studies provide information on the nature and extent of association between any two pairs of metric characters correlation between any two pairs of traits due to genotypic and environmental causes. Grain yield is the end product of interactions of many factors knows as contributing components hence it is complex traits. Understanding of the interaction of characters among themselves and with the environment has been of great use in the plant breeding, the genotypic correlation between different characters of the plant could arise because of linkage relationship. In general, the genotypic correlation coefficient values were higher than the phenotypic values. This indicated that how much of phenotypic correlation 
coefficient is influenced by environment. In the present study, all possible correlation coefficient at genotypic and phenotypic levels among 16 traits themselves and with grain yield/plant was estimated. The phenotypic correlation coefficient has been discussed below:

In the present investigation the grain yield per plant was positively and significantly associated with 1000 grain weight, number of grains per ear, number of spikelets per spike, biological yield, days to maturity, ear weight, ear length, harvest index, peduncle length. The results found in the present investigation were agreement with Ali et al., (2008). Kahrizi et al., (2010) were found high correlations among the plant height, leaf dry weight, stem dry weight, spike dry weigh, Mohibullah et al., (2011) were found spike length showed positive significant correlation with a number of spikelets per spike and grain yield per plant. Whereas plant height, canopy temperature, numbers of ears per plant, number of effective tillers per plant, days to $50 \%$ heading, and chlorophyll content had shown positive non-significant, and RWC had shown negative non-significant on grain yield. The present findings are in contradiction with Kashif and Khaliq (2004), Ali et al., (2008), Dharmendra and Singh (2015) who observed that grain yield per plant showed highly significant positive correlation with number of productive tillers per plant, number of spikelets per spike and number of grains per spike and significant positive correlation with spike length.

\section{Path coefficient analysis}

The correlation coefficient between characters exhibits the relationship existing between pairs of characters. But, a dependent character is an interaction of product of many mutually associated component characters and change in any one component will disturb the whole network of cause and effect system. The path coefficient analysis, a statistical device developed by Wright (1921), which takes into account the cause and effect relationship between the variables. The path coefficient partitions the association into direct and indirect effects via other the relative importance of causal factors involved. This is simply standardized regression analysis. Path diagram facilitates the understanding the nature of cause and effect system. The path analysis suggested by Dewey and Lu (1959) helps to resolve these correlations further and lighter on the way in which component traits contribute towards specifically identifying important traits.

In the present investigations path coefficient analysis has been performed at genotypic and phenotypic levels taking yield as a dependent variable. In general genotypic direct and indirect effects were slightly higher in magnitude as compared to the phenotypic effects. Biological yield revealed the highest direct effect on grain yield followed by a number of ears per plant, harvest index, ear length, 1000-grain weight, days to maturity, canopy temperature, peduncle length, canopy temperature. However, number of spikelets per spike, RWC, number of grains per ear, plant height, days to $50 \%$ heading, ear weight, and number of effective tillers per plant had a negative direct effect on grain yield. The results found in the present investigation were in agreement with the findings of (Khokhar et al., 2010) where they observed that the highest positive direct effect on yield was exhibited by days to maturity followed by days to heading. Fikre et al., (2015) studied sixty-four bread wheat genotypes and observed that grain weight, spike length, number of grains per plant and number of spikelets per spike had positive direct effects on grain yield. Majority of indirect effects of various independent traits via other traits were extremely low of either sign. There were only 
a few characters which had higher to moderate positive indirect effects. The indirect effects of 1000- grain weight, number of spikelets per spike, biological yield, days to maturity, ear weight, ear length, harvest index, peduncle length. Hence these indirect effects should also be kept in mind while selection for better yield.

\section{References}

Ali Y, Atta BM, Akhter J, Monneveux P and Lateef Z. 2008. Genetic Variability, Correlation and Path Coefficient for Eight Metric Traits in Wheat (Triticum aestivum L.) germplasm, Pakistan journal of botany 40: 2087-2097

Dewey JR and Lu KH. 1959. A correlation and path coefficient analysis of components of crested wheat grass seed production. Agronomy journal 51: 515518

Dharmendra S and Singh KN. 2015. Variability analysis for yield and yield attributes of bread wheat under salt affected condition, http://www. shigen. nig.ac. jp/ewis /article /html/80/article.html

Fikre G, Alamerew S and Tadesse Z. 2015. Path coefficient and correlation studies of yield and yield associatied traits ijn bread wheat (Triticum aestivum L.) genotypes at kulumsa Agricultural Research center, South East Ethopia. Food science and quality management 38: $14-23$

Fisher RA and Yates F. 1963. Statistical tables for biological, agricultural and medical research. Oliver and Boyd, London
Food and Agriculture Organization of the United Nations. 2015. FAO statistical division, http/faostat.fao.org $\quad\left(3^{\text {rd }}\right.$ August, 2015)

Kahrizi D, Cheghamirza K, Kakaei M, Mohammadi R and Ebadi A. 2010. Heritability and genetic gain of some morphophysiological variables of durum wheat (Triticum turgidum var. durum). African journal of biotechnology 9: 4687-4691

Kashif M and Khaliq I. 2004. Heritability, Correlation and Path Coefficient Analysis for Some Metric Traits in W heat. International journal of agriculture and biology 6: 138-142

Khokhar MI, Hussain M, Zulkiffal M, Ahmad $\mathrm{N}$ and Sabar W. 2010. Correlation and path analysis for yield and yield contributing characters in wheat (Triticum aestivum L.). African journal of plant science 4: 464 -466

Miller PA, Williams JC, Robinson HF and Comstock RE. 1958. Variance and covariance in Cotton. Agronomy journal 50: 126-131

Mohibullah M, Rabbani MA, Jehan S, Zakiullah, Amin A and Farullah G. 2011. Studied Correlation on different Wheat Genotypes (Triticum aestivum L.) Pakistan journal of botany 43: 27172720

Reynolds MP, Hobbs PR, Ortiz R, Pietragalla $J$ and Braun HJ. 2008. Wheat improvement: Highlights from an expert symposium. CIMMYT

Wright S. 1921. Correlation and Causation. Journal of agricultural science 20: 557587

\section{How to cite this article:}

Rana Saha, Hausila Prasad Singh, Sandeep Kumar, Md. Ekram Hussain and Shukla R. S. 2018. Wheat Genotypes under Restricted Irrigated Conditions. Int.J.Curr.Microbiol.App.Sci. 7(06): 3251-3263. doi: https://doi.org/10.20546/ijcmas.2018.706.383 